\title{
Electric and Magnetic Properties of Sputter Deposited $\mathrm{BiFeO}_{3}$ Films
}

\author{
N. Siadou, ${ }^{1}$ I. Panagiotopoulos, ${ }^{1}$ N. Kourkoumelis, ${ }^{2}$ T. Bakas, ${ }^{3}$ \\ K. Brintakis, ${ }^{4,5}$ and A. Lappas ${ }^{4}$ \\ ${ }^{1}$ Department of Materials Science and Engineering, University of Ioannina, 45110 Ioannina, Greece \\ ${ }^{2}$ Department of Medical Physics, University of Ioannina, 45110 Ioannina, Greece \\ ${ }^{3}$ Department of Physics, University of Ioannina, 45110 Ioannina, Greece \\ ${ }^{4}$ Institute of Electronic Structure and Laser, Foundation for Research and Technology-Hellas, Vassilika Vouton, 711 10 Heraklion, Greece \\ ${ }^{5}$ Department of Physics, Aristotle University of Thessaloniki, 54124 Thessaloniki, Greece
}

Correspondence should be addressed to I. Panagiotopoulos; ipanagio@cc.uoi.gr

Received 4 October 2013; Accepted 17 October 2013

Academic Editor: Tung-Ming Pan

Copyright (c) 2013 N. Siadou et al. This is an open access article distributed under the Creative Commons Attribution License, which permits unrestricted use, distribution, and reproduction in any medium, provided the original work is properly cited.

\begin{abstract}
Polycrystalline $\mathrm{BiFeO}_{3}$ films have been magnetron sputter deposited at room temperature and subsequently heat-treated ex situ at temperatures between 400 and $700^{\circ} \mathrm{C}$. The deposition was done in pure Ar atmosphere, as the use of oxygen-argon mixture was found to lead to nonstoichiometric films due to resputtering effects. At a target-to-substrate distance $d=2^{\prime \prime}$ the $\mathrm{BiFeO}_{3}$ structure can be obtained in larger range process gas pressures (2-7 mTorr) but the films do not show a specific texture. At $d=6^{\prime \prime}$ codeposition from $\mathrm{BiFeO}_{3}$ and $\mathrm{Bi}_{2} \mathrm{O}_{3}$ has been used. Films sputtered at low rate tend to grow with the (001) texture of the pseudocubic $\mathrm{BiFeO}_{3}$ structure. As the film structure does not depend on epitaxy similar results are obtained on different substrates. A result of the volatility of $\mathrm{Bi}, \mathrm{Bi}$ rich oxide phases occur after heat treatment at high temperatures. $\mathrm{A} \mathrm{Bi}_{2} \mathrm{SiO}_{5}$ impurity phase forms on the substrate side, and does not affect the properties of the main phase. Despite the deposition on amorphous silicon oxide substrate weak ferromagnetism phenomena and displaced loops have been observed at low temperatures showing that their origin is not strain. $\mathrm{Ba}, \mathrm{La}, \mathrm{Ca}$, and $\mathrm{Sr}$ doping suppress the formation of impurity phases and leakage currents.
\end{abstract}

\section{Introduction}

Recently there is a revival of the interest in magnetoelectric materials for novel multifunctional devices [1] and spintronic [2] applications. $\mathrm{BiFeO}_{3}(\mathrm{BFO})$ is both ferroelectric $\left(T_{C}=1100 \mathrm{~K}\right)$ and antiferromagnetic $\left(T_{N}=643 \mathrm{~K}\right)$ at room temperature, and thus it is very promising for such applications [3]. Pulsed laser deposition [4-6] and sputtering [7-10] are standard methods to deposit BFO films. Magnetron sputtering is a very reproducible and easily controlled deposition technique that can be used to prepare heterostructures combining different types of layers. However, when magnetrons are used to prepare binary oxide materials, severe resputtering of the film during deposition can alter its composition [11]. In order to find the conditions which lead to formation of the crystalline BFO phase sputtering at different substrate-to-target distances and process gas pressures have been tested. Here, we present a study of magnetron sputtered deposition of polycrystalline $\mathrm{BiFeO}_{3}$ films in view of their use as exchange-biasing layers.

\section{Experimental}

Polycrystalline $\mathrm{BFO}$ and iron oxide films have been magnetron sputter deposited at room temperature using a MANTIS deposition system from a commercial $\mathrm{BiFeO}_{3}\left(2^{\prime \prime}\right.$ and $\left.3^{\prime \prime}\right), \mathrm{Bi}_{2} \mathrm{O}_{3}\left(2^{\prime \prime}\right)$, and $\mathrm{Fe}_{3} \mathrm{O}_{4}\left(3^{\prime \prime}\right)$ targets of Kurt J. Lesker. The substrates are placed opposite to the sputtering targets (on axis). Pure (5N) Ar was used as a process gas. Three different methods have been applied: (i) deposition from a $2^{\prime \prime}$ BFO at a substrate-to-target distance of $2^{\prime \prime}$, (ii) low rate deposition from a $2^{\prime \prime} \mathrm{BFO}$ at a substrate-to-target distance of $6^{\prime \prime}$, and 


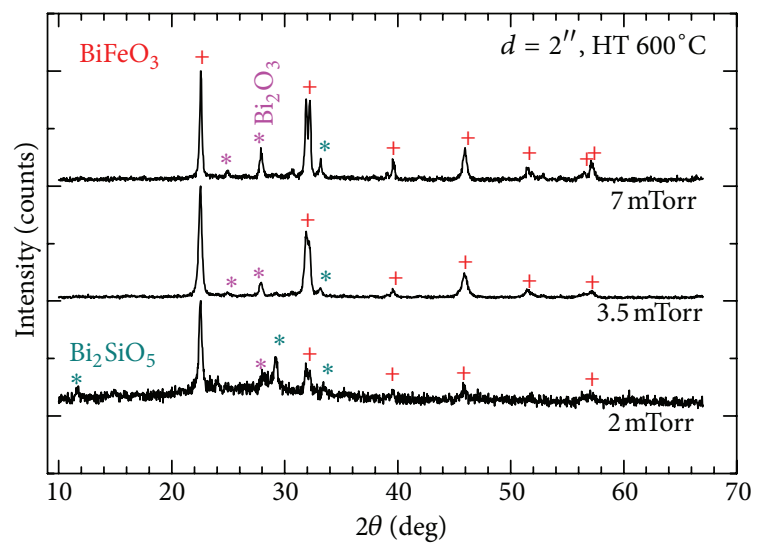

FIGURE 1: XRD patterns of bismuth ferrite films deposited under different sputtering gas pressures at a distance $d=2^{\prime \prime}$.

(iii) codeposition from a $\mathrm{BFO}\left(3^{\prime \prime}\right)$ and $\mathrm{Bi}_{2} \mathrm{O}_{3}\left(2^{\prime \prime}\right)$ targets at a distance of $6^{\prime \prime}$. In order to achieve doping by elements as $\mathrm{Ba}, \mathrm{La}, \mathrm{Sr}$, and $\mathrm{Ca}$, corresponding oxide powders have been added either on the $\mathrm{BiFeO}_{3}$ or on the $\mathrm{Bi}_{2} \mathrm{O}_{3}$ target for heavy or low doping, respectively. The BFO films have been sputter deposited and subsequently heat-treated ex situ at temperatures between 400 and $700^{\circ} \mathrm{C}$. The X-ray diffraction (XRD) diagrams were collected with a Bruker D8 Advance Diffractometer. The magnetic measurements were performed with a Lake Shore vibrating sample magnetometer (VSM) and Quantum Design SQUID magnetometer. The microstructure was investigated with a JEOL JSM-5600 Scanning Electron Microscope. The leakage currents have been measured by a RT66B test system of Radiant technologies.

\section{Optimization of the Deposition Conditions}

In Figure 1, the XRD patterns of films deposited under different sputtering gas pressures at a distance $d=2^{\prime \prime}$ are presented. The applied power is $100 \mathrm{~W}$ RF. In all cases, $\mathrm{BiFeO}_{3}$ is the main phase, and the stoichiometry (determined by EDX) does not vary considerably, but it is close to $\mathrm{Bi}_{47} \mathrm{Fe}_{53} \mathrm{O}_{x}$. More specifically, the atomic percent of $\mathrm{Bi}$ was determined to be 46.2, 47.5, and 47.1, for 2, 3.5, and 7 mTorr of Ar gas, respectively.

There is presence of bismuth oxide, mainly at higher Ar pressure, and $\mathrm{Bi}_{2} \mathrm{SiO}_{5}$ at lower Ar pressures. The former is a result of the volatility of $\mathrm{Bi}$ and the decomposition of the bismuth ferrite phase. If additional oxygen is used as a reactive gas, then the films are severely Bi deficient (EDX shows $\mathrm{Bi}_{40} \mathrm{Fe}_{60} \mathrm{O}_{x}$ ) and the $\mathrm{BiFeO}_{3}$ phase does not form. This may be attributed to resputtering as a result of oxygen anions bombardment of the substrate [11]. The $\mathrm{Bi}_{2} \mathrm{SiO}_{5}$ phase forms on the substrate side, as suggested by the presence of $\mathrm{Si}$, and does not affect the properties of the main phase.

This can be proven by examining grazing incidence XRD patterns acquired at different incidence angles. The $\mathrm{Bi}_{2} \mathrm{SiO}_{5}$ peak intensity increases with the angle of incidence (Figure 2).

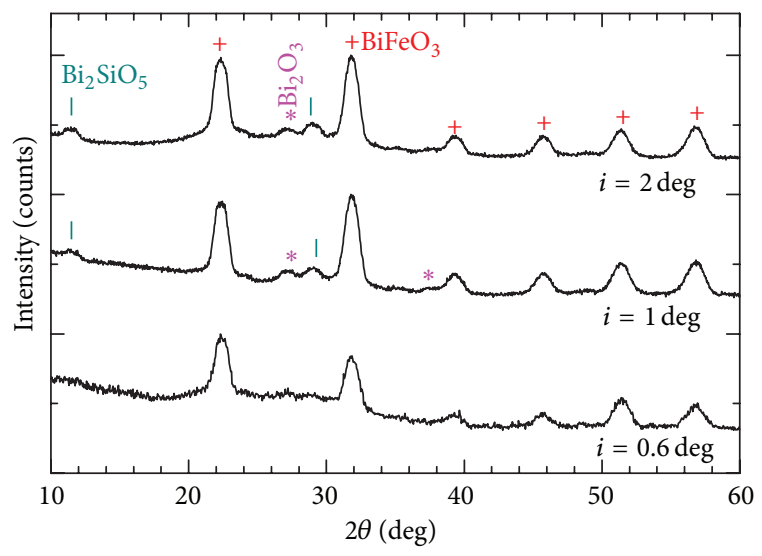

FIGURE 2: Grazing incidence XRD patterns of BFO films measured under different incidence angles. $\mathrm{BiFeO}_{3}$ peaks are indicated by crosses, those of $\mathrm{Bi}_{2} \mathrm{SiO}_{5}$ by lines, and those of $\mathrm{Bi}_{2} \mathrm{O}_{3}$ by stars.

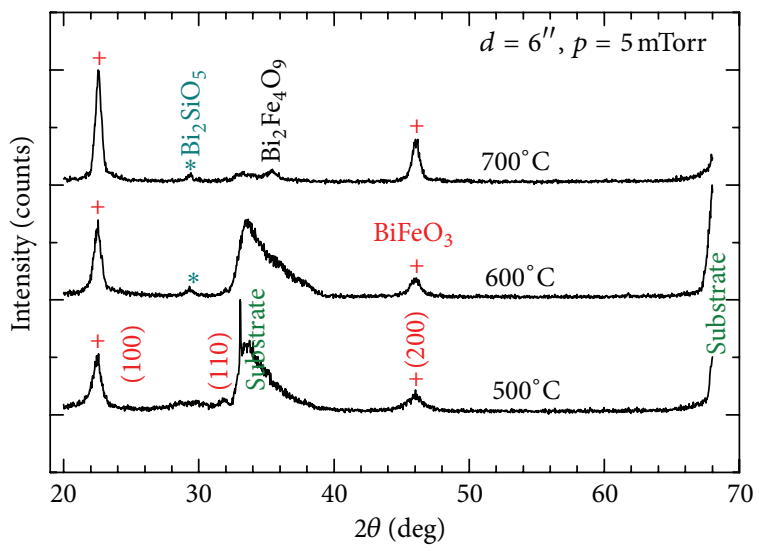

FIGURE 3: XRD patterns of bismuth ferrite films deposited Ar gas pressure $p=5$ mTorr at a distance $d=6^{\prime \prime}$ on oxidized Si wafers. The films show (100) texture of the pseudocubic unit cell which corresponds to the (012) of R3c.

In Figure 3 the XRD patterns of $20 \mathrm{~nm}$ thick heat-treated films deposited at $d=6^{\prime \prime}$ at Ar pressure of 5 mTorr with deposition rate $0.03 \mathrm{~nm} / \mathrm{min}$. Similar results are obtained for films deposited at Ar pressure of $2 \mathrm{mTorr}(0.25 \mathrm{~nm} / \mathrm{min})$. After ex situ heat treatment at $500^{\circ} \mathrm{C}$, the $\mathrm{BiFeO}_{3}$ phase with (100) texture of the pseudocubic unit cell appears. This corresponds to the (012) of rhombohedral phase (space group R3c) which is the bulk unit cell, resulting from the symmetry reduction due to the ferroelectric and accompanying distortions. The optimum heat-treatment temperature is $600^{\circ} \mathrm{C}$ while heat treatment at higher temperatures leads to the formation of the undesired phases as silicate, bismuth oxide, and in some cases other ferrites as $\mathrm{Bi}_{2} \mathrm{Fe}_{4} \mathrm{O}_{9}$.

The degree of texture was estimated by profile refinement of the XRD patterns using the WinPLOTR package (Figure 4). The March preferred orientation function was used. The March parameter estimated to 0.22 which gives an average grain misalignment consistent with the rocking curves around the (001) pseudocubic reflection. In general, it was found that, under these conditions, the stoichiometry 


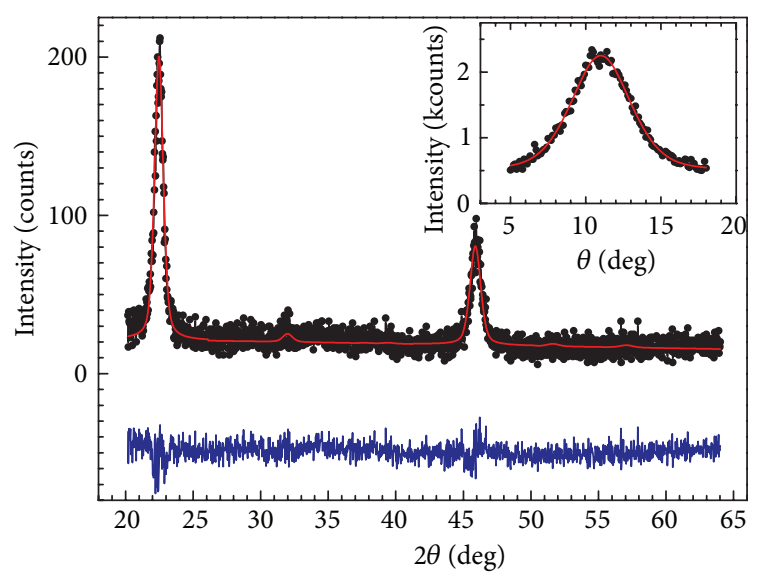

FIGURE 4: Rietveld fit of an XRD pattern of bismuth ferrite films deposited Ar gas pressure $p=2$ mTorr at a distance $d=6^{\prime \prime}$ and heat-treated at $500^{\circ} \mathrm{C}$. The line at the bottom shows the difference between the theoretical and the experimental line. The inset shows a rocking curve around the (001) reflection.

and $\mathrm{BiFeO}_{3}$ formation thereof was very sensitive to target usage partially due to magnetic field limitations of the $2^{\prime \prime}$ source. Therefore, we have chosen to use a $3^{\prime \prime} \mathrm{BiFeO}_{3}$ and enhance $\mathrm{Bi}$ content by codepositing from $2^{\prime \prime} \mathrm{Bi}_{2} \mathrm{O}_{3}$ target. The rates were $1.38 \mathrm{~nm} / \mathrm{min}$ and $0.25 \mathrm{~nm} / \mathrm{min}$, respectively. This small stoichiometry correction is crucial in reproducibly achieving the $\mathrm{BiFeO}_{3}$ phase. In thicker films $(260 \mathrm{~nm})$ prepared under the same conditions, there is a strong presence of the cubic $\mathrm{Bi}_{25} \mathrm{FeO}_{40}$ phase. The impurity phases can be leached by $10 \%$ diluted $\mathrm{HNO}_{3}$ for 30 minutes.

\section{Microstructure and Domains}

SEM studies have been performed to heat-treated BFO films which have been field cooled under $2 \mathrm{kOe}$ from above the Néel temperature. Thus the formation of the ferroelectric domains is expected to be influenced by the coupling to the existing AF domains, due to the multiferroic nature of $\mathrm{BFO}$ that implies coupling between the two types of order [12]. The ferroelectric domain structure can be revealed by SEM using the brightness contrast between antiparallel ferroelectric domains on an unmetalized polar crystal surface [13-15]. Low accelerating voltage (down to $2 \mathrm{kV}$ ) and small beam current are used, in order to avoid severe charge accumulation on the insulating surface of the sample.

In $\mathrm{BFO}$, the ferroelectric polarization can point along any of the eight directions defined by the four diagonals of the pseudocubic perovskite unit cell (with two antiparallel polarities for each direction). Between these eight possible different polar domains in $\mathrm{BiFeO}_{3}\left[\begin{array}{lll} \pm 1 & \pm 1 & \pm 1\end{array}\right]$ there are three possible types of ferroelectric domain walls $71^{\circ}$, $109^{\circ}$, and $180^{\circ}$ degrees typical of rhombohedral crystals. Different types of domains can be favored on the growth conditions [16]. These domains are stripe-like, and their width scales with film thickness. Very different irregular domain morphology has been observed in thin epitaxial films
[13]. In our case, the use of Si wafers covered with amorphous Si oxide layers excludes the possibility of strain appearance as relaxation mechanism in the morphology of domains. On the contrary, the polycrystalline nature and grain boundaries create complex multidomain structures.

SEM images of $125 \mathrm{~nm}$ thick films prepared at $d=$ $2^{\prime \prime}$ show that the films consist of large $20-100 \mu \mathrm{m}$ islandlike grains (Figure 5). The domain patterns are cloud-like irregular with size 3-7 $\mu \mathrm{m}$. This complex domain structure may be attributed to the fact that, due to the existence of isolated islands, a unique global minimum cannot be achieved. Films prepared at $d=6^{\prime \prime}$ look homogenous at larger areas and are also characterized by a fine mosaic-like domain structure in the range of $10 \mu \mathrm{m}$ (Figure 6).

\section{Weak Ferromagnetism Phenomena}

Weak ferromagnetism phenomena have been observed long ago in antiferromagnetic (AF) fine particles and have been explained as a result of unbalanced magnetic moments of the two magnetic sublattices due to their finite size [17]. The presence of uncompensated surface spins leads to anomalous magnetic properties, such as large moments, coercivities, and hysteresis loop shifts. The antiferromagnetic ordering of $\mathrm{BiFeO}_{3}$ is G-type that is, each spin is surrounded by six antiparallel spins on the nearest Fe neighbors. This ordering should give rise to magnetically compensated interfaces which is not favorable to exchange biasing. Thus surface magnetic heterogeneities, complexity of the underlying BFO film and nanoscale domain wall features, are crucial for the development of exchange biasing [14]. In sufficiently small particles the reduced coordination of surface spins can cause a fundamental change in the magnetic order throughout the particle [18]. In this case, a clear distinction between surface and bulk spin contributions to the total magnetic moment cannot be done. In AF thin films these phenomena are expected to dominate at low film thickness due to the increased contribution of the surfaces. The hysteresis loops at low temperatures (Figure 7) show ferromagnetic contributions, coercivity of $150 \mathrm{Oe}$, and a small exchange biasing evidenced by the vertical displacement of the loops, which is maximal $(120 \mathrm{Oe})$ for the $87 \mathrm{~nm}$ film. The high field slope corresponds to the canting of the AF moments which is maximal for the $43 \mathrm{~nm}$ thin film. The remanence values are low and must be attributed to both bulk and surface contributions.

These different contributions become clearer in the magnetization versus temperature plots (Figure 8). The surface contribution is enhanced below $20 \mathrm{~K}$. The thickness dependence implies that there is a surface contribution of $6.4 \mu \mathrm{emu} / \mathrm{cm}^{2}$ and volume contribution of $1.16 \mathrm{emu} / \mathrm{cm}^{3}$ at $5 \mathrm{~K}$ which become $4.3 \mu \mathrm{emu} / \mathrm{cm}^{2}$ and $0.7 \mathrm{emu} / \mathrm{cm}^{3}$, respectively, at $300 \mathrm{~K}$. These values range from 0.065 to $0.04 \mu_{B} / \mathrm{Fe}$ atom. As the magnetization values are higher for thinner films they must be attributed to surface contributions and should not be confused with some weaker contributions due to intrinsic mechanisms which could reflect an underlying competition between antiferromagnetic and ferromagnetic 


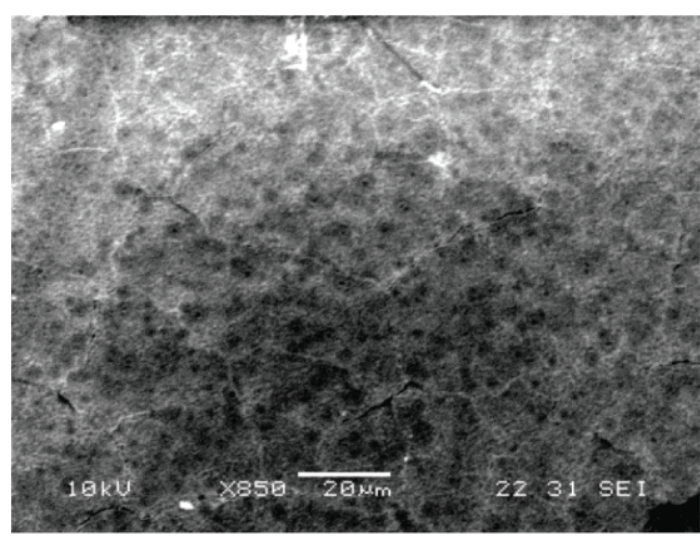

(a)

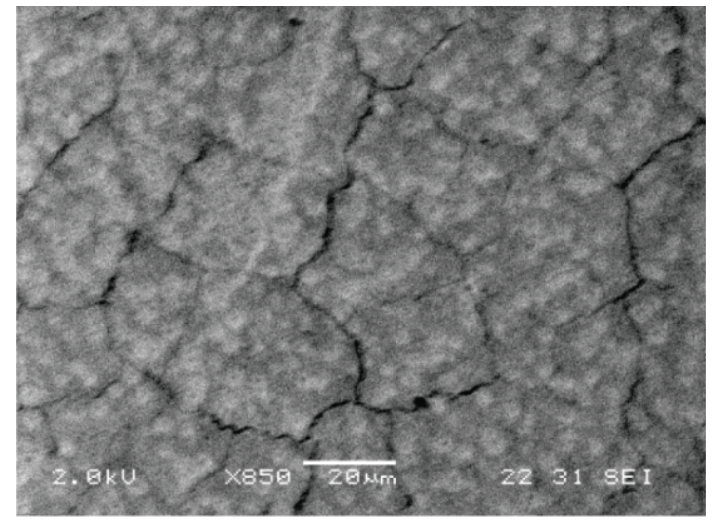

(b)

FIGURE 5: SEM images of a $125 \mathrm{~nm}$ thick $\mathrm{BiFeO}_{3}$ film prepared at a source-to-substrate distance of $2^{\prime \prime}$. (a) was obtained with accelerating voltage of $10 \mathrm{kV}$. (b) was obtained with $2.5 \mathrm{kV}$. Large $(\sim 50 \mu \mathrm{m})$ grains are observed along with smaller features related to the ferroelectric domain structure.

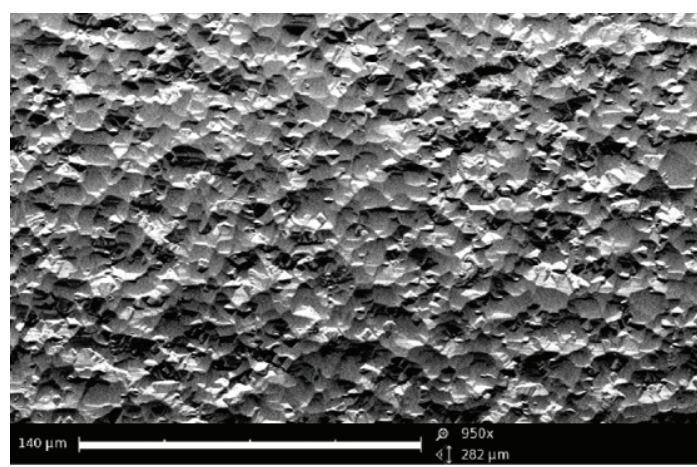

(a)

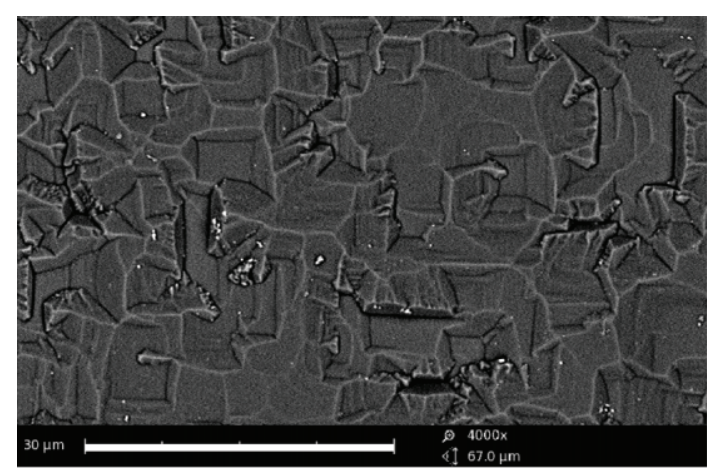

(b)

Figure 6: SEM images of an $87 \mathrm{~nm}$ thick $\mathrm{BiFeO}_{3}$ film prepared at a source-to-substrate distance of 6".

interactions and the appearance of spin-glass state in the intermediate temperature range. Similar thickness dependence has been reported for spin-glass phenomena observed in compressively strained $\mathrm{BiFeO}_{3}$ films from 19 to $114 \mathrm{~nm}$ epitaxially grown on $\mathrm{LaAlO}_{3}$ [19]. The observation of similar behavior in our films deposited on the amorphous silicon oxide layers shows that strain mechanisms that have been revoked are not the only possible causes leading to the appearance of low temperature ferromagnetic contributions.

\section{Effects of Doping}

The XRD patterns of the $\mathrm{Bi}_{1-x} \mathrm{Ba}_{x} \mathrm{FeO}_{3}$ series are shown in Figure 9. Ba doping suppresses the formation of impurity phases. In Figure 10, the variation of unit cell with Ba content is shown. The data are compared with the simple Vegard's law for a solid solution, extrapolating between the end members $\mathrm{BiFeO}_{3}$ and $\mathrm{BaFeO}_{3}$ (dashed line). The continuous line is a fit based on a simple geometrical relation for the size of the perovskite cell with the ionic radii as free parameters, yielding $1.35 \mathrm{~nm}, 1.42 \mathrm{~nm}$, and $1.45 \mathrm{~nm}$ for $\mathrm{Bi}^{3+}, \mathrm{Ba}^{2+}$, and oxygen, respectively. These values differ from those tabulated by Shannon [20] $(1.03 \mathrm{~nm}, 1.35 \mathrm{~nm}$, and $1.40 \mathrm{~nm}$, resp.). The systematic increase of the cell constant with doping shows that $\mathrm{Ba}$ enters the main perovskite phase.

Low doping range is more interesting as it does not affect the ferroelectric properties, and it is sufficient to suppress the leakage currents. A compilation of the leakage current measurements, under different doping, is shown in Figure 11. Reduction of leakage is believed to occur because of the reduced oxygen vacancy which stabilizes the oxygen octahedral [21]. However, the abrupt enhancement of current at a threshold voltage can be attributed to an electronic localization-delocalization transition through band-filling control since oxygen vacancy distribution should be continuously varied by sweeping the applied voltage [22]. In general, the effect of substitutions is not limited to the "chemical pressure" due to ionic-radius mismatch with respect to $\mathrm{Bi}$ (minimal for $\mathrm{Ca}, \mathrm{La}$, moderate for $\mathrm{Sr}$, and highest for $\mathrm{Ba}$ ) but also to the effects to the stereochemically active $\left(6 s^{2}\right)$ lonepair activity. In this case, the best results have been obtained for Sr and Ca substitutions. 


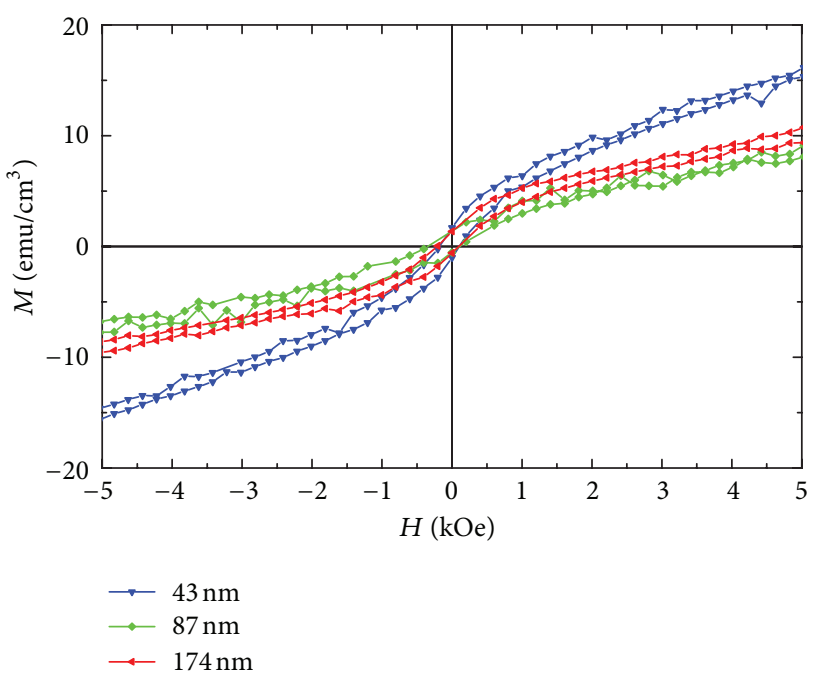

Figure 7: Hysteresis curves at $5 \mathrm{~K}$ after field cooling under a field of $70 \mathrm{kOe}$. Only the low field range is shown.

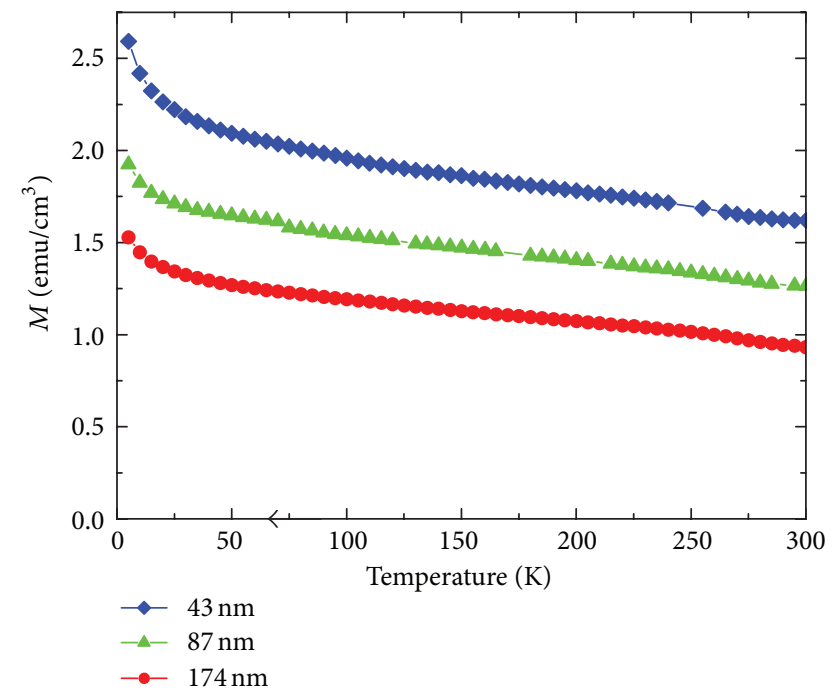

Figure 8: Magnetization as a function of temperature for BFO films of different thickness. The measurements have been done upon warming from $5 \mathrm{~K}$ to $300 \mathrm{~K}$ in the presence of a 100 Oe field after cooling under a field of $70 \mathrm{kOe}$.

\section{Conclusion}

Polycrystalline BFO films have been grown by RF magnetron sputtering on different substrates. The formation of the BFO depends very sensitively on sputtering conditions and heattreatment temperature. In general, it was found that, when the substrate is placed close to the target $d=2^{\prime \prime}$, the formation of $\mathrm{BFO}$ is more stable, that is, the phase can be obtained within a wide range of conditions but the films do not show a specific crystallographic texture, in contrast to those sputtered at $d=6^{\prime \prime}$. As the films have been grown on the amorphous oxide layers of silicon wafers, it is proven that texture can be achieved without epitaxy of $20 \mathrm{~nm}$ films and furthermore that the low temperature weak ferromagnetism phenomena

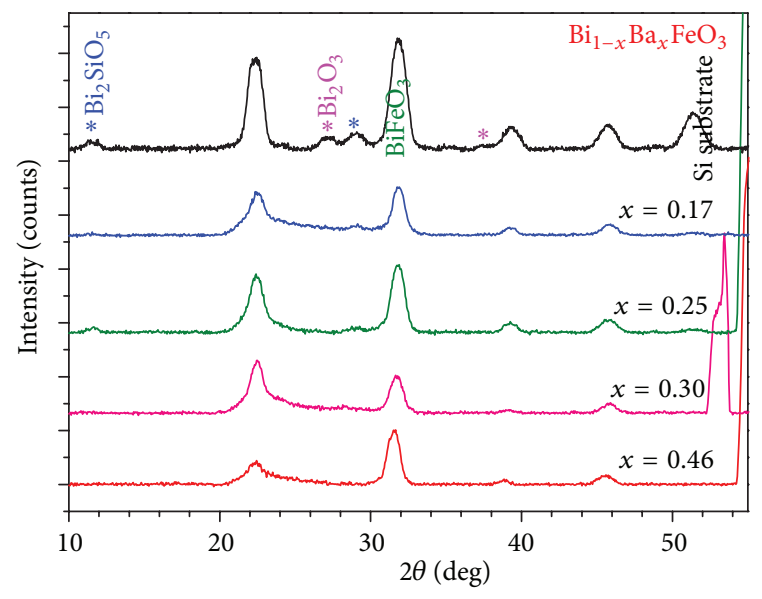

FIGURE 9: Grazing incidence XRD patterns $\mathrm{Bi}_{1-x} \mathrm{Ba}_{x} \mathrm{FeO}_{3}$ films.

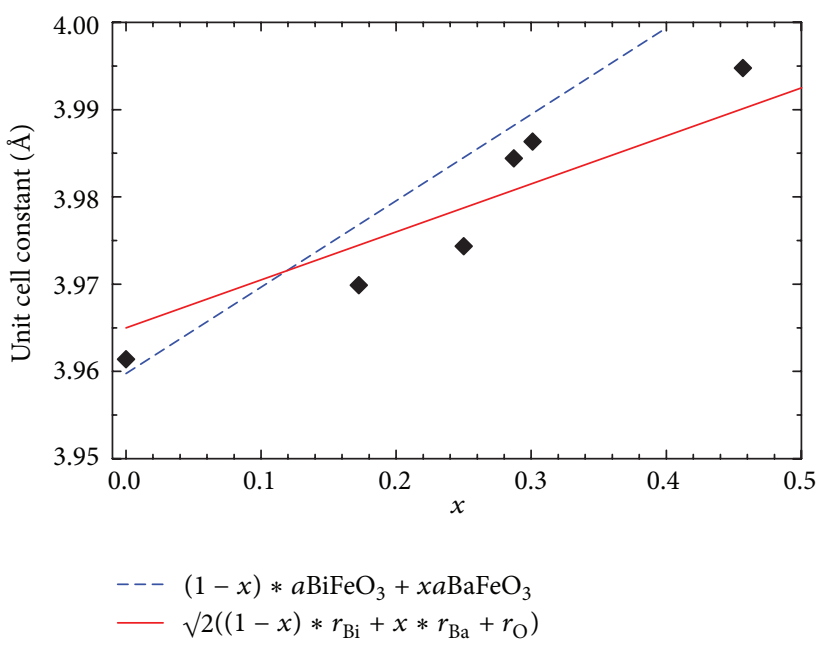

FIgURE 10: Pseudocubic unit cell parameter of $\mathrm{Bi}_{1-x} \mathrm{Ba}_{x} \mathrm{FeO}_{3}$ films.

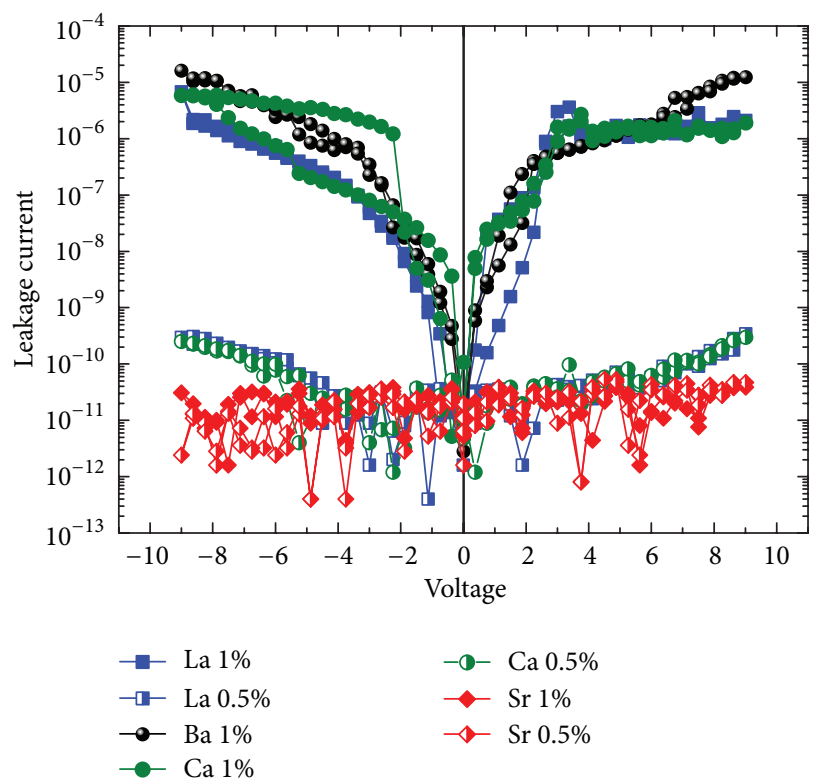

FIGURE 11: Leakage current across $90 \mathrm{~nm}$ thick doped BFO films (i.e., the maximum voltage corresponds to $100 \mathrm{kV} / \mathrm{cm}$ ). 
are not related to epitaxial strains. Doping suppresses the formation of impurity phases and leakage currents.

\section{Acknowledgments}

This research has been cofinanced by the European Union (European Social Fund-ESF) and Greek national funds through the Operational Program "Education and Lifelong Learning" of the National Strategic Reference Framework (NSRF)-Research Funding Program: Heracleitus-II. Investing in knowledge society through the European Social Fund. The authors would like to thank the authors John Kafegidakis and Ruud Bernsen at Phenom-World BV for recording some of the SEM images.

\section{References}

[1] C.-W. Nan, M. I. Bichurin, S. Dong, D. Viehland, and G. Srinivasan, "Multiferroic magnetoelectric composites: historical perspective, status, and future directions," Journal of Applied Physics, vol. 103, no. 3, Article ID 031101, 2008.

[2] M. Bibes and A. Barthélémy, "Towards a magnetoelectric memory," Nature Materials, vol. 7, pp. 425-426, 2008.

[3] G. Catalan and J. F. Scott, "Physics and applications of bismuth ferrite," Advanced Materials, vol. 21, no. 24, pp. 2463-2485, 2009.

[4] V. R. Palkar, J. John, and R. Pinto, "Observation of saturated polarization and dielectric anomaly in magnetoelectric $\mathrm{BiFeO}_{3}$ thin films," Applied Physics Letters, vol. 80, no. 9, pp. 1628-1630, 2002.

[5] F. Zavaliche, S. Y. Yang, T. Zhao et al., "Multiferroic $\mathrm{BiFeO}_{3}$ films: domain structure and polarization dynamics," Phase Transitions, vol. 79, no. 12, pp. 991-1017, 2006.

[6] H. X. Lu, J. L. Zhao, J. R. Sun, J. Wang, and B. G. Shen, "Ferroelectric domain structure of the $\mathrm{BiFeO}_{3}$ film grown on different substrates," Physica B, vol. 406, no. 3, pp. 305-308, 2011.

[7] Y. Li, T. Sritharan, S. Zhang, X. He, Y. Liu, and T. Chen, "Multiferroic properties of sputtered $\mathrm{BiFeO}_{3}$ thin films," Applied Physics Letters, vol. 92, no. 13, Article ID 132908, 2008.

[8] R. Y. Zheng, X. S. Gao, Z. H. Zhou, and J. Wang, "Multiferroic $\mathrm{BiFeO}_{3}$ thin films deposited on $\mathrm{SrRuO}_{3}$ buffer layer by rf sputtering," Journal of Applied Physics, vol. 101, no. 5, Article ID 054104, 2007.

[9] Z. Chaodan, Z. Duanming, Y. Jun et al., "Synthesis and ferroelectric properties of $\mathrm{BiFeO}_{3}$ thin films grown by sputtering," Integrated Ferroelectrics, vol. 94, no. 1, pp. 23-30, 2007.

[10] C. Ternon, J. Thery, T. Baron, C. Ducros, F. Sanchette, and J. Kreisel, "Structural properties of films grown by magnetron sputtering of $\mathrm{BiFeO}_{3}$ target," Thin Solid Films, vol. 515, no. 2, pp. 481-484, 2006.

[11] J. M. Grace, D. B. McDonald, M. T. Reiten, J. Olson, R. T. Kampwirth, and K. E. Gray, "The effect of oxidant on resputtering of Bi from Bi-Sr-Ca-Cu-O films," The Journal of Vacuum Science and Technology, vol. 10, no. 4, p. 1600, 1991.

[12] Y.-H. Chu, L. W. Martin, M. B. Holcomb, and R. Ramesh, "Controlling magnetism with multiferroics," Materials Today, vol. 10, no. 10, pp. 16-23, 2007.

[13] S. Zhu and W. Cao, "Imaging of 180 ferroelectric domains in $\mathrm{LiTaO}_{3}$ by means of scanning electron microscopy," Physica Status Solidi A, vol. 173, 1999.
[14] G. Catalan, H. Béa, S. Fusil et al., "Fractal dimension and size scaling of domains in thin films of multiferroic $\mathrm{BiFeO}_{3}$," Physical Review Letters, vol. 100, no. 2, Article ID 027602, 2008.

[15] L. W. Martin, Y.-H. Chu, M. B. Holcomb et al., "Nanoscale control of exchange bias with $\mathrm{BiFeO}_{3}$ thin films," Nano Letters, vol. 8, no. 7, pp. 2050-2055, 2008.

[16] Y. B. Chen, M. B. Katz, X. Q. Pan et al., "Ferroelectric domain structures of epitaxial (001) $\mathrm{BiFeO}_{3}$ thin films," Applied Physics Letters, vol. 90, no. 7, Article ID 072907, 2007.

[17] A. H. Morrish, The Physical Principles of Magnetism, John Wiley \& Sons, New York, NY, USA, 1965.

[18] H. Ohldag, A. Scholl, F. Nolting et al., "Correlation between exchange bias and pinned interfacial spins," Physical Review Letters, vol. 91, no. 1, Article ID 017203, 2003.

[19] C.-J. Cheng, C. Lu, Z. Chen et al., "Thickness-dependent magnetism and spin-glass behaviors in compressively strained $\mathrm{BiFeO}_{3}$ thin films," Applied Physics Letters, vol. 98, no. 24, Article ID 242502, 2011.

[20] R. D. Shannon, "Revised effective ionic radii and systematic studies of interatomic distances in halides and chalcogenides," Acta Crystallographica A, vol. 32, pp. 751-767, 1976.

[21] H. Ishiwara, "Impurity substitution effects in $\mathrm{BiFeO}_{3}$ thin filmsm-dashFrom a viewpoint of FeRAM applications," Current Applied Physics, vol. 12, no. 3, pp. 603-611, 2012.

[22] C.-H. Yang, J. Seidel, S. Y. Kim et al., "Electric modulation of conduction in multiferroic Ca-doped $\mathrm{BiFeO}_{3}$ films," Nature Materials, vol. 8, no. 6, pp. 485-493, 2009. 

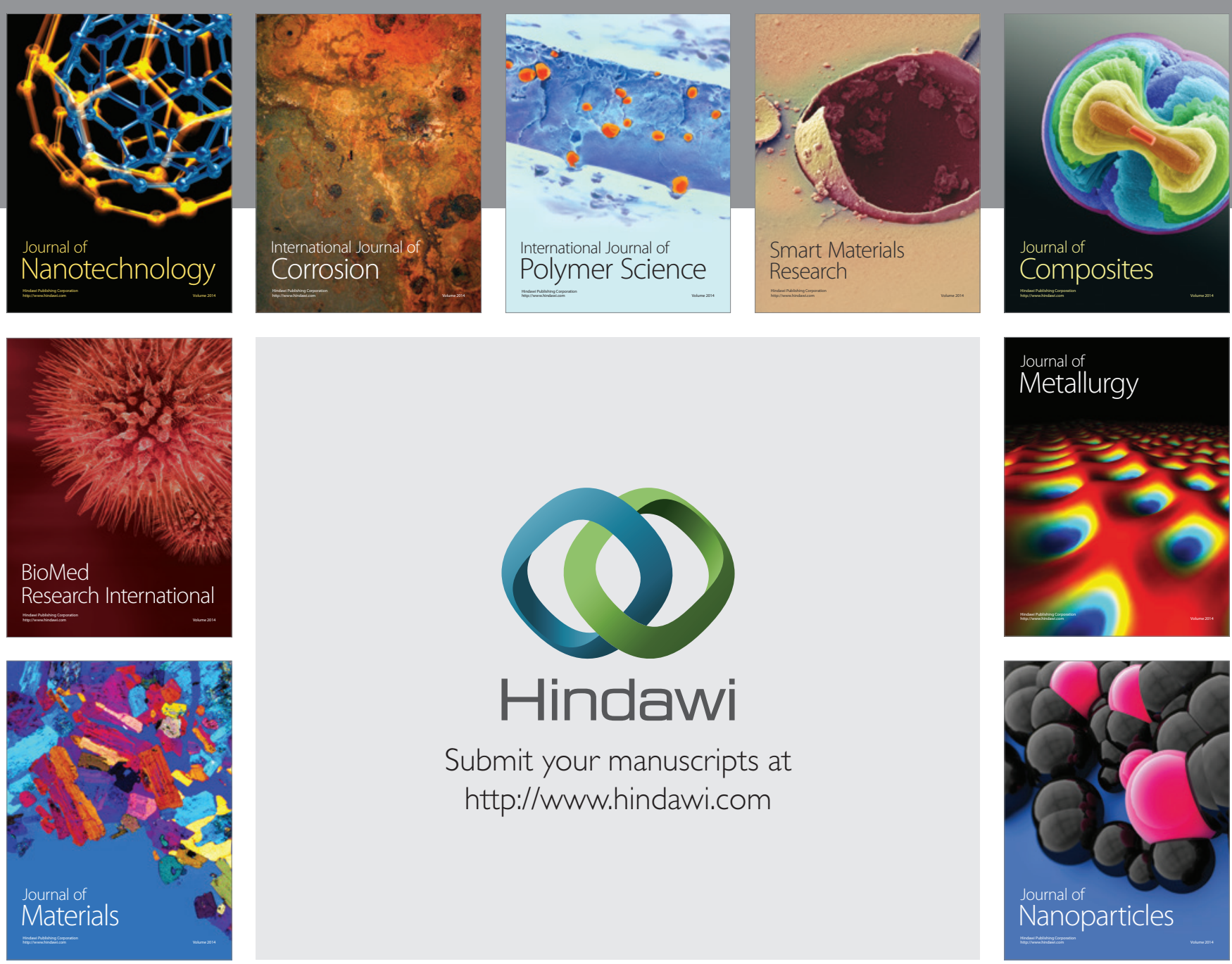

Submit your manuscripts at http://www.hindawi.com
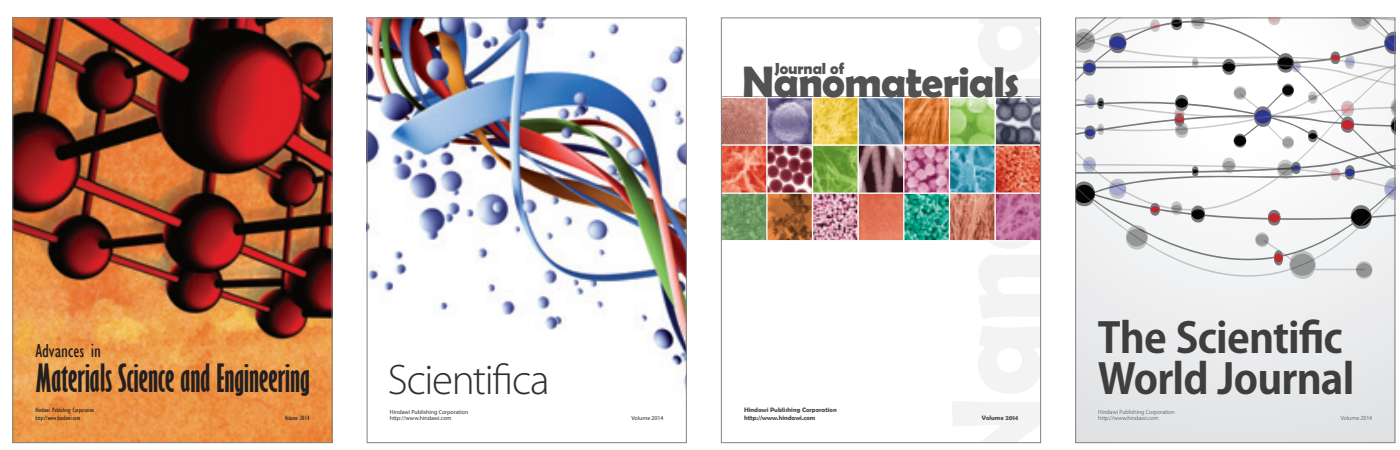

\section{The Scientific World Journal}
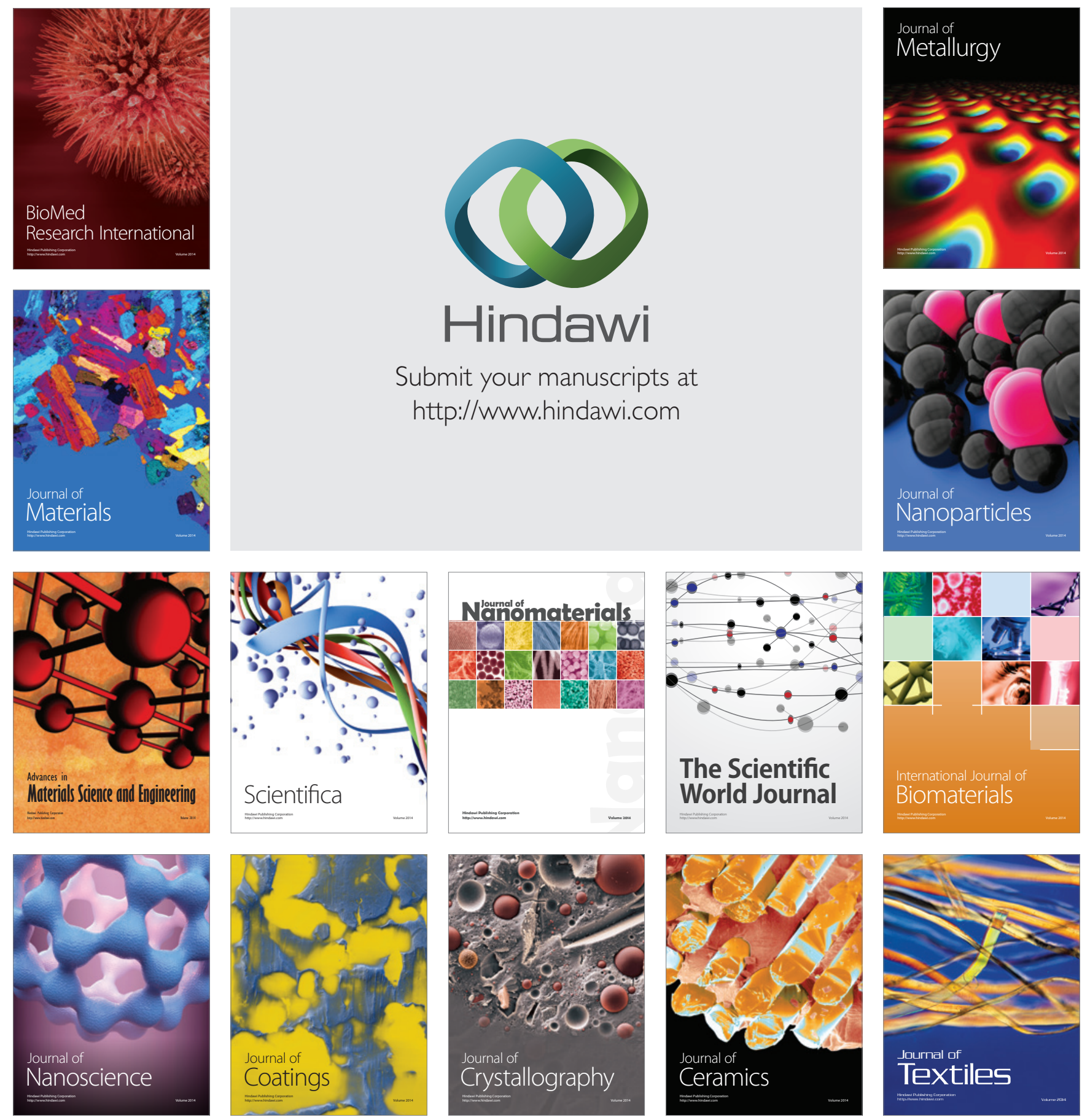